\title{
Design and Study the Effect of Switching in Microstrip Patch Antenna for Cognitive Radio Applications
}

\author{
Gurpreet Kumar and Gursharanjeet Singh Kalra \\ School of Electronics and Communication Engineering, Lovely Professional \\ University Phagwara - 144411, Punjab, India; \\ gurpreet.14632@lpu.co.in, gursharanjeet.singh@lpu.co.in
}

\begin{abstract}
Design of microstrip antenna having switches for accomplishing frequency reconfigurability is proposed. The proposed design is having six resonating frequency bands between $3.34 \mathrm{GHz}$ to $3.67 \mathrm{GHz}$ which were achieved by using different switching configurations. Switches were assumed as ideal so as to study the simulated results of the antenna .The antenna design was made on the FR4 substrate having height of $1.6 \mathrm{~mm}$. The overall size of the antenna is $39 \times 40 \mathrm{~mm}^{2}$. Simulated results are utilized to validate performance of antenna. The radiation pattern, return loss and measured bandwidth are presented in the paper. Simulated results presented in this paper make the antenna are appropriate for applications such as cognitive radio which requires sensing of spectrum to use the bandwidth in an optimal manner.
\end{abstract}

Keywords: Cognitive, PIN Diodes, Reconfigurable, Switching

\section{Introduction}

Recently the use of RF switches in microsrtip patch antenna for achieving reconfigurability has been a great area of research. The parameters, for example, $f$ radiation pattern, frequency and polarization are changed to satisfy the application requirements. These antennas can be used in application like cognitive radio which requires the process of spectrum sensing to use the spectrum wisely $\frac{1,8.9}{\text {. }}$. There are other techniques that have been applied as well to achieve a desired operation in an antenna such as, physical size changing, addition of multiple layers and gaps $^{14}$, using stacked patches ${ }^{\frac{12}{2}}$, inserting slits and changing the substrate material ${ }^{13}$. However these tequniques can increase the size of the antenna significantly which will make it difficult for these antennas to fit into small devices ${ }^{2-4}$. Further design optimization techniques needs to be employed on these antennas to decrease the effective length and width of antenna ${ }^{3-6}$.

There have been multiple innovative techniques that have been used to achieve the reconfigurable operation in an antenna such as use of PIN diodes, varactor diodes to act as switches $\frac{10,11}{1}$. To change the current distribution in the slot FET components have also been used which actually alters the effective length of the antenna. The use of micro-electro-mechanical system to improve the accuracy in switching has also been widely employed in the designs, but this increases the cost and circuit complexity in the design ${ }^{5-7}$.

This paper aims to design and analyze microstrip antenna and study the effect of switching on the parameters of the antenna for example, gain, return loss,

*Author for correspondence 
directivity and VSWR of the antenna. The different switching configurations are presented in this paper. The switches are acting as ideal so small conducting slits were used to show the ON position of the switch while doing the simulation on CST Microwave Studio.

\section{Antenna Configuration and Design}

We will be discussing about the design structure of the antenna in this section. Figure 1 and 2 is showing the overall geometry of designed antenna. Antenna was designed using FR4 substrate having thickness $1.6 \mathrm{~mm}$ and tangent loss 0.025 . The size of the patch is $19.5 \times 20 \mathrm{~mm}^{2}$. Microstrip line feed technique was used in this design and width of the feed line is $4.5 \mathrm{~mm}$. Slot is cut at the bottom of the antenna having area of $1 \times 20 \mathrm{~mm}^{2}$. There are four switches that have been inserted in the ground plane at different position to observe the behavior of the antenna under different logical conditions of the switches. By turning on the switches at different instances six resonating frequency bands have been simulated. Omni directional and bidirectional radiation patterns have been observed at different resonating frequencies of the antenna and are presented later in this paper. The antenna initially resonates at $3.63 \mathrm{GHz}$ with full ground plane and when the slot was used at the bottom it produced different resonating frequencies.

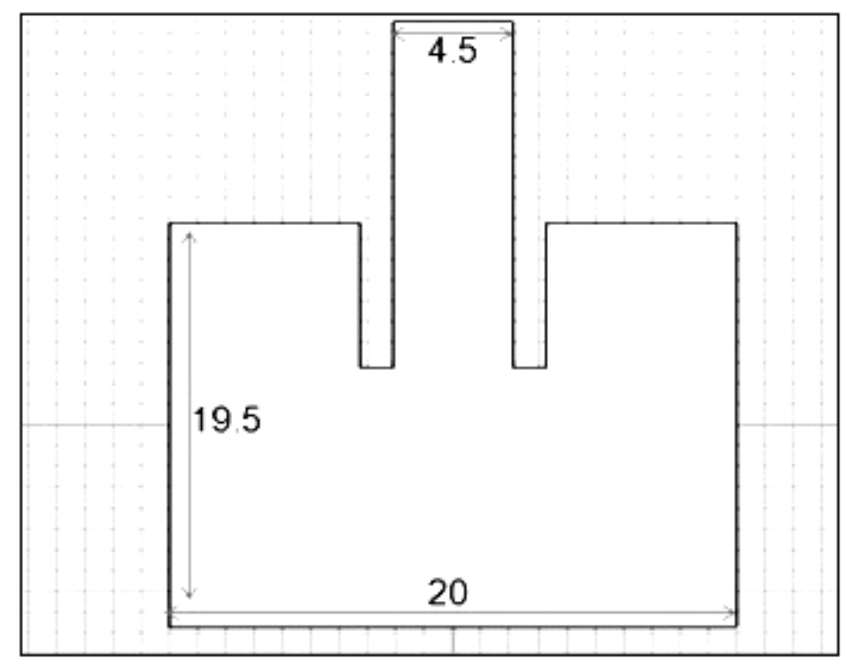

Figure 1. Top View.

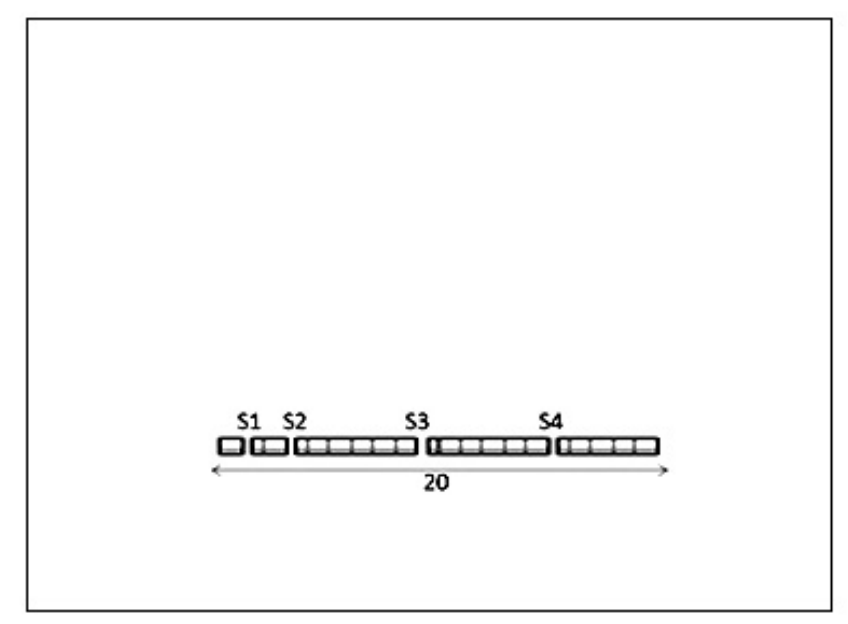

Figure 2. Bottom View.

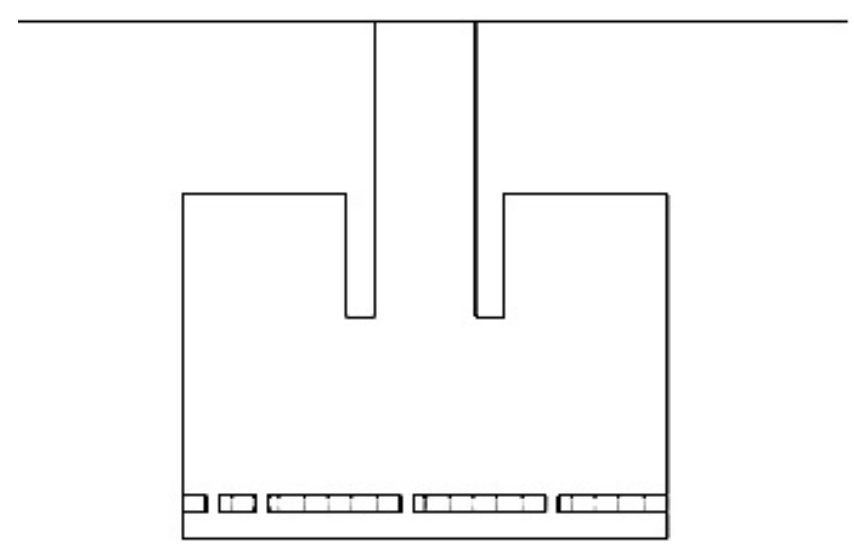

Figure 3. Antenna top view and switches at the bottom.

\section{Results and Discussions}

\subsection{Simulated Results of Initial Design with No Slot}

The Figure 4 is showing return loss plot of the initial design structure having no slot cut in the ground plane. The antenna was resonating at $3.63 \mathrm{GHz}$ and was having a return loss of $-21.85 \mathrm{~dB}$. The VSWR of the design was also simulated its value was 1.17 . The radiation pattern of the initial design was omni-directional having a gain of $3.31 \mathrm{~dB}$. The design was then modified and a slot was cut in the bottom ground plane of the antenna to introduce multiple resonating modes in the antenna by varying the overall effective size of the antenna. 


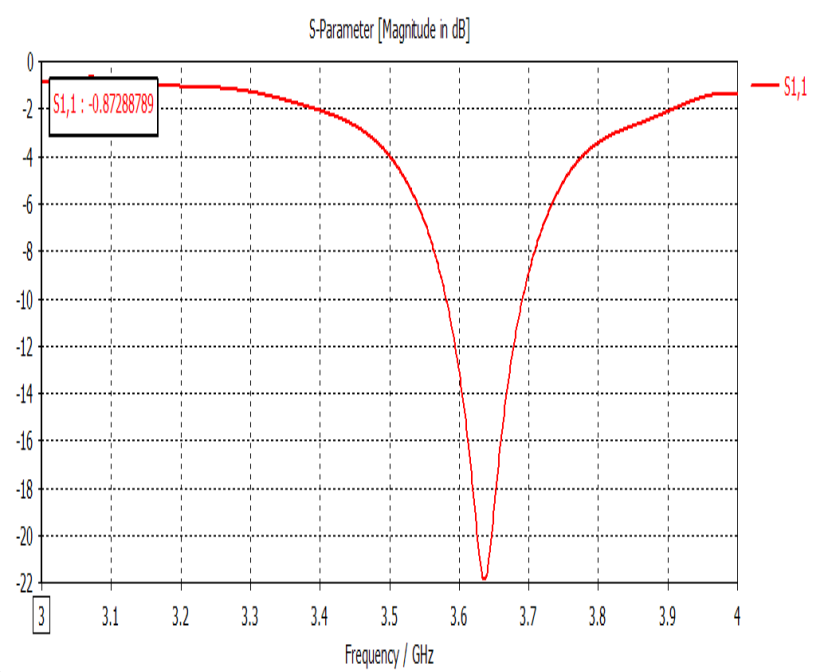

Figure 4. Return loss plot of initial design.

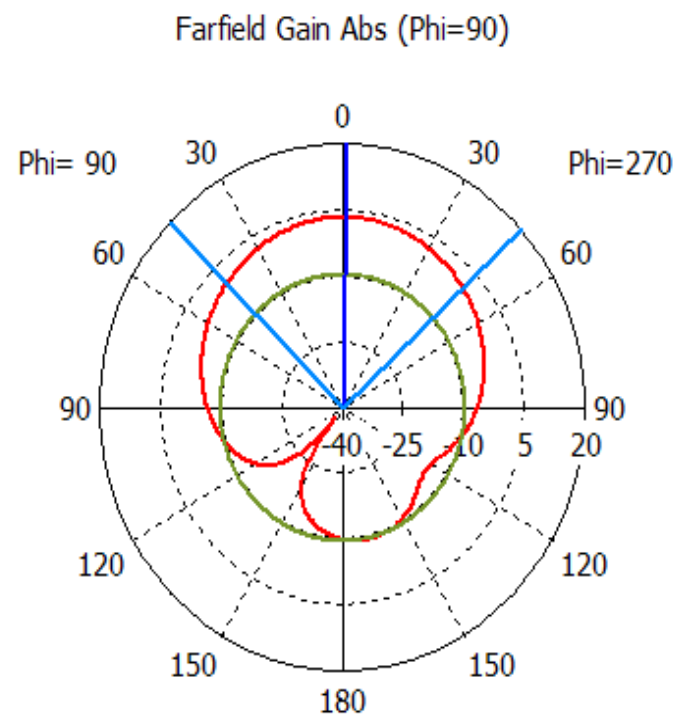

Theta / Degree vs. dB

Figure 5. Radiation pattern of initial design.

\subsection{Simulated Results by Cutting Slot in Bottom Ground Plane and by Introducing Switches}

By inserting the slot and introducing four small switches in bottom ground plane of designed antenna, multiple resonant frequency bands were observed. Four switches were positioned optimally in the slot. The different switch configurations are shown on the Table 1. When S1 is on the antenna is resonating at $3.34 \mathrm{GHz}$ having a return loss of $-39.72 d B$. Similarly by switching on switches S2, S3 and $\mathrm{S} 4$ antenna was resonating at $3.45 \mathrm{GHz}, 3.60 \mathrm{GHz}$ and $3.52 \mathrm{GHz}$ having return loss of $-44.27 \mathrm{~dB},-39.19 \mathrm{~dB},-37.31$ $\mathrm{dB}$ respectively. The radiation pattern at all frequencies as having same orientation with different gains. Similarly the VSWR of the design was having value below 1.5 at all resonating frequencies. The percentage bandwidth at all resonating frequencies was lying in the range of $3.11 \%$ to $4.31 \%$ and overall bandwidth ratio of 1.1:1 was measured from the simulated results.

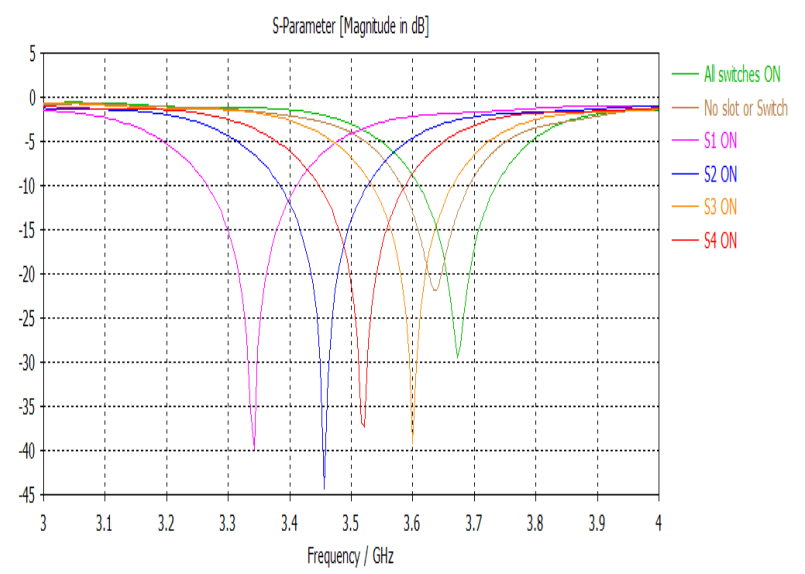

Figure 6. Different switching configurations showing return loss of the design.

Radiation patterns at various resonating frequencies can be seen in fig.7. All the radiation patterns are having similar radiation pattern orientation except the gain at each resonating frequency is different. The maximum gain of $4.04 \mathrm{~dB}$ was measured at resonating frequency of $3.45 \mathrm{GHz}$ and minimum gain of $3.31 \mathrm{~dB}$ was measured at resonating frequency of $3.63 \mathrm{GHz}$. At all other frequencies the gain of the antenna lies in this range.

Table 1. Switch configurations showing simulated return loss and measured bandwidth

\begin{tabular}{|l|c|c|c|}
\hline $\begin{array}{l}\text { Switch } \\
\text { Configurations }\end{array}$ & Frequency(GHz) & $\begin{array}{c}\text { Return } \\
\text { Loss(dB) }\end{array}$ & $\begin{array}{c}\text { Measured } \\
\text { bandwidth }\end{array}$ \\
\hline $\begin{array}{l}\text { No slot or } \\
\text { Switch }\end{array}$ & 3.63 & -21.85 & $3.11 \%$ \\
\hline $\begin{array}{l}\text { All switches } \\
\text { ON }\end{array}$ & 3.67 & -29.39 & $3.39 \%$ \\
\hline S1 ON & 3.34 & -39.72 & $4.31 \%$ \\
\hline S2 ON & 3.45 & -44.27 & $4.10 \%$ \\
\hline S3 ON & 3.60 & -39.19 & $3.65 \%$ \\
\hline S4 ON & 3.52 & -37.31 & $3.97 \%$ \\
\hline
\end{tabular}


Farfield Gain Abs (Phi=90)

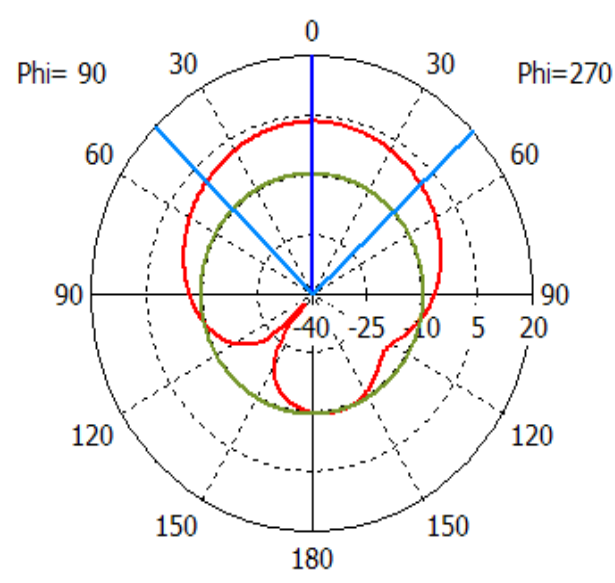

Theta / Degree vs. dB

(a)

Farfield Gain Abs (Phi=90)

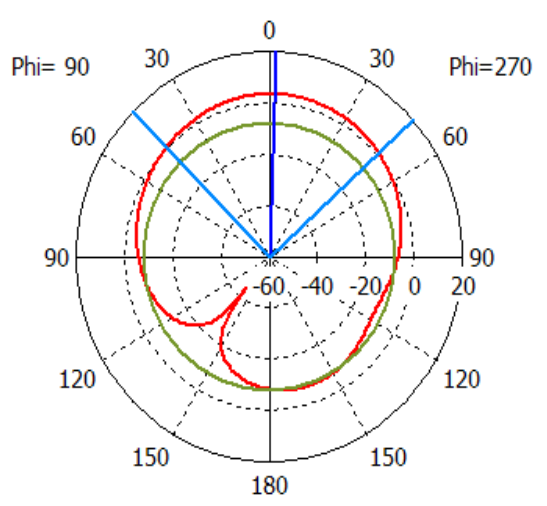

Theta / Degree vs. dB

(b)

Farfield Gain Abs (Phi=90)

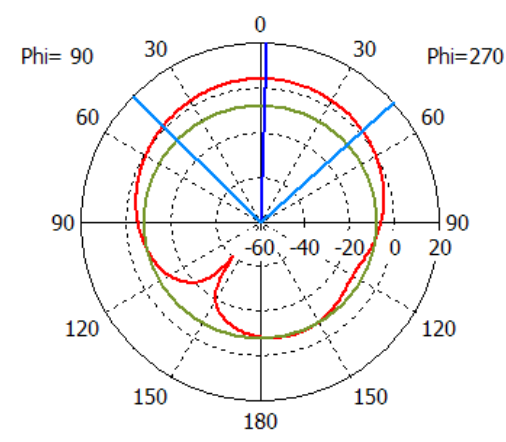

Theta / Degree vs. dB

(c)
Farfield Gain Abs (Phi=90)

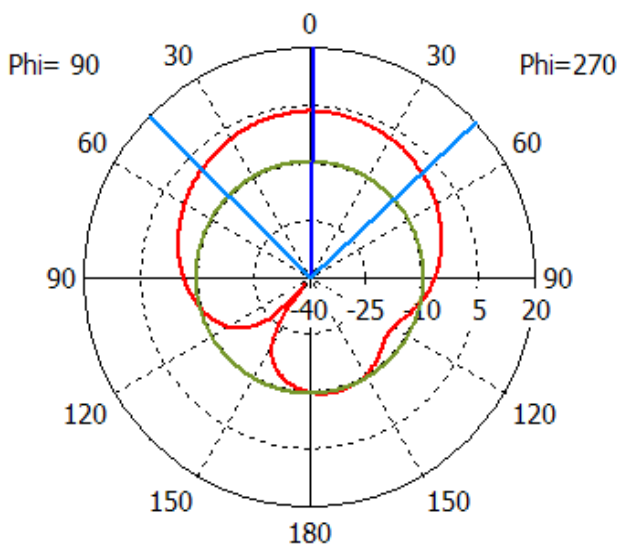

Theta / Degree vs. dB

(d)

Farfield Gain Abs (Phi=90)

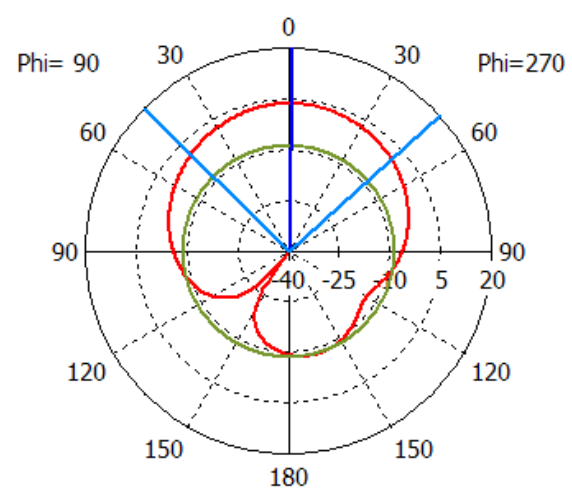

Theta / Degree vs. dB

(e)

Figure 7. Radiation pattern at (a) $3.67 \mathrm{GHz}$ (b) $3.342 \mathrm{GHz}$ (c) $3.45 \mathrm{GHz}(\mathrm{d}) 3.60 \mathrm{GHz}$ (e) $3.52 \mathrm{GHz}$.

\section{Conclusion}

Effect of switching in microstrip patch antenna has been presented in this paper. Multiple resonant frequency bands within bandwidth ratio of 1.1:1 are shown. It has been observed that by changing the switching configuration in the design the effective size of the radiating patch changes and antenna resonates at different frequency. RF components and MEMs can be used to fabricate the design's switching configuration. 


\section{References}

1. Mazid H. Frequency-reconfigurable microstrip patch-slot antenna. IEEE Antennas and Wireless Propagation Letters. 2013; 12:218-220.

2. Nilavalan R. A reconfigurable wideband and multiband antenna using dual-patch elements for compact wireless devices. IEEE transactions on antennas and propagation. January 2012; 60(1):36-43.

3. Harish Rajagopalan, Joshua Kovitz. Frequency reconfigurable wideband E-shaped patch antenna: design, optimization and measurements. IEEE; 2012.

4. Yingsong Li, Wenxing. Integrated dual-purpose narrow/ ultra-wide band antenna for cognitive radio applications. IEEE; 2012.

5. M.Lai T Wu. Design of reconfigurable antennas based on L-shaped slot and PIN diodes for compact wireless devices. IET Microwave Antennas Propagation. 2009; 3(1): Doi: 10.1049 .

6. Yufeng Yu. An electrically small frequency reconfigurable antenna with a wide tuning range. IEEE Antennas and Wireless Propagation Letters. 2011; 10:103-6.

7. Abdel-Fattah Sheta, Samir F. Mahmoud. A widely tunable compact patch antenna. IEEE Antennas and Wireless Propagation Letters. 2008; 7:40-2.

8. Ghaith Mansour, Peter S. Hall. Tunable Slot-loaded Patch antenna for cognitive radio. 2012 Loughborough Antennas and Propagation Conference: UK. November 2012. p. 12-13.

9. Yong Cai,Y. Jay Guo. A frequency reconfigurable printed yagi-uda dipole antenna for cognitive radio applications. IEEE Transactions on Antennas and Propagation. June 2012; 60(6): 2905 - 12.

10. Han TY, Huang CT. Reconfigurable monopolar patch antenna. Electronics Letters. 4th February 2010; 46(3): 199-200.

11. Hossein Hadidian Moghadam, Ali Mirkamli. Using printed dipole antenna and PIN diodes for wideband frequency reconfiguration. 2010 Loughborough Antenna and Propagation Conference. 8-9 November 2010.UK.

12. Muhamud S, Ali MT. A reconfigurable microstrip antenna with a slotted patch at dual frequency. 2118 th international symposium on wireless communication system. Aachen.

13. Farnaz Mirzamohammadi, Javad Nourinia. A novel dual-wideband monopole-like microstrip antenna with controllable frequency response. IEEE Antennas Wireless Propagation Letters. 2012; 11:289-92.

14. Billy Wu, Michal Okoniewski. A novel scheme for realizing a microstrip antenna with switchable circular polarization. 6th European Conference on Antennas and Propagation. 2011. 